\title{
FATORES ASSOCIADOS À REALIZAÇÃO DO EXAME CITOPATOLÓGICO EM MULHERES PROFISSIONAIS DO SEXO
}

\author{
FACTORS ASSOCIATED WITH THE \\ PERFORMANCE OF CYTOPATHOLOGICAL \\ EXAMINATION IN FEMALE SEX WORKERS
}

\section{FACTORES ASOCIADOS A LA REALIZACIÓN DEL EXÁMEN CITOPATOLÓGICO EN MUJERES PROFESIONALES DEL SEXO}

\author{
Rosilane de Lima Brito Magalhães ${ }^{1}$ \\ Braulio Vieira de Sousa Borges ${ }^{2}$ \\ Vanessa Moura Carvalho de Oliveira ${ }^{3}$ \\ Giselle Mary Ibiapina Brito ${ }^{3}$ \\ Ana Karysa Alves Resende ${ }^{4}$ \\ Elucir Gir
}

Como citar este artigo: Magalhães RLB, Borges BVS, Oliveira VMC, Brito GMI, Resende AKA, Gir E. Fatores associados à realização do exame citopatológico em mulheres profissionais do sexo. Rev baiana enferm. 2018;32:e25931.

Objetivos: estimar a prevalência autorreferida da realização do exame citopatológico do colo do útero em mulheres profissionais do sexo e investigar a associação entre a realização desse teste e os dados socioeconômicos e comportamentais. Método: estudo analítico transversal, com 416 mulheres profissionais do sexo, em uma capital do nordeste brasileiro entre janeiro de 2014 e fevereiro de 2015. Resultados: a prevalência autorreferida de realização do exame citopatológico do colo do útero foi de 47,8\%. Houve associações entre a realização do teste e a renda ( $p=0,001)$, a escolaridade $(p=0,001)$, a religião $(p=0,001)$, a forma de acesso ao serviço $(p=0,003)$ e o uso de contraceptivo $(\mathrm{p}=0,003)$. Conclusão: mulheres profissionais do sexo apresentaram baixa prevalência para realização do exame citopatológico do colo do útero, fato que ocorre especialmente entre as mulheres mais empobrecidas, pouco escolarizadas e com dificuldade de acesso aos serviços de saúde.

Descritores: Teste de Papanicolaou. Profissionais do sexo. Mulheres. Prevalência. Prevenção secundária. Atenção primária à saúde.

Objectives: to estimate the self-reported prevalence forperforming the cervical cytopathological examination in female sex workers and investigate the association between its performance and the socioeconomic and behavioral data. Method: a cross-sectional study with 416 female sex workers in a capital city of the Brazilian Northeast between

\footnotetext{
Enfermeira. Doutora em Enfermagem Fundamental. Professora Permanente da Pós-graduação em Enfermagem da Universidade Federal do Piauí. Teresina, Piauí, Brasil.

Enfermeiro. Mestre em Enfermagem. Enfermeiro da Secretaria de Saúde do Distrito Federal. Braślia, Distrito Federal, Brasil. braulitos89@hotmail.com

Enfermeira. Integrante do Grupo de Estudos em Doenças Infecciosas e outros agrafos da Universidade Federal do Piauí. Teresina, Piauí, Brasil.

Enfermeira. Coordenadora da Estratégia Saúde da Família. Secretaria Municipal de Saúde de Boa Hora. Teresina, Piauí, Brasil.

Enfermeira. Doutora em Enfermagem. Professor Titular da Escola de Enfermagem de Ribeirão Preto, Universidade de São Paulo. Coordenadora da Rede de Enfermagem Nacional em AIDS e DST. Ribeirão Preto, São Paulo, Brasil.
} 
January 2014 and February 2015. Results: the self-reported prevalence for performing the cervical cytopathological examination was $47.8 \%$. There were associations between the performance of the test and income $(p=0.001)$, schooling $(p=0.001)$, religion ( $p=0.001)$, service access $(p=0.003)$, and contraceptive use $(p=0.003)$. Conclusion: female sex workers presented a low prevalence for performing the cervical cytopathological examination, especially among the poorest women, with little education, and with difficulty accessing health services.

Keywords: Pap smear test. Sex workers. Women. Prevalence. Secondary prevention. Primary bealth care.

Objetivos: estimar la prevalencia autorreferida de la realización del examen citopatológico del cuello uterino en mujeres profesionales del sexo e investigar la asociación entre la realización de ese test y los datos socioeconómicos y comportamentales. Método: estudio analítico transversal, con 416 mujeres profesionales del sexo, en una capital del noreste brasileño entre enero de 2014 y febrero de 2015. Resultados: la prevalencia autorreferida de la realización del examen citopatológico del cuello uterino fue del 47,8\%. Hubo asociaciones entre la realización del test y la renta $(p=0,001)$, la escolaridad $(p=0,001)$, la religión $(p=0,001)$, la forma de acceso al servicio $(p=0,003) y$ el uso de anticonceptivos $(p=0,003)$. Conclusión: mujeres profesionales del sexo presentaron baja prevalencia para la realización del examen citopatológico del cuello uterino, hecho que ocurre especialmente entre mujeres más empobrecidas, poco escolarizadas y con dificultad de acceso a los servicios de salud.

Descriptores: Test de Papanicolaou. Profesionales del sexo. Mujeres. Prevalencia. Prevención secundaria. Atención primaria a la salud.

\section{Introdução}

O câncer do colo do útero é causa comum de mortalidade em mulheres, havendo cerca de 270.000 casos por ano em todo o mundo ${ }^{(1)}$. No Brasil, as principais causas de morte feminina têm sido pelo câncer de mama e colorretal, seguido do câncer do colo uterino. Ressalta-se que, no país, ocorreu redução acentuada da mortalidade no período de 1990 a 2015 para -33,9\%, especificamente em Rondônia, Roraima, Alagoas, em todos os estados das regiões Sul e Sudeste e em quase todos os estados da região Centro-Oeste ${ }^{(2)}$.

Os fatores de risco mais importantes para o desenvolvimento do câncer do colo do útero são, dentre outros: infecção pelo Papiloma Vírus Humano (HPV), início precoce da atividade sexual, multiplicidade de parceiros sexuais, baixa condição socioeconômica, uso prolongado de contraceptivos orais ${ }^{(3)}$. Assim, Mulheres Profissionais do Sexo (MPS) têm fatores de risco elevados para neoplasias do colo uterino, pois possuem multiplicidade de parceiros sexuais, apresentando um risco aumentado de infecção por HPV ${ }^{(4)}$.

Um estudo realizado na China demonstrou que ser acometido pelo HPV foi mais prevalente entre as MPS (61,90\%) quando comparadas com o restante da população $(21,00 \%)(\mathrm{p}<0,01)$ e que os principais fatores de risco associados foram a idade da primeira relação sexual (OR 0,69; IC95\% 0,49-0,99) e a pós-menopausa (OR 2,92; IC95\% 1,09-7,80) $(\mathrm{p}<0,05)^{(5)}$.

Nessa perspectiva, a realização do exame citopatológico do colo do útero é uma ferramenta importante para o diagnóstico de alterações patológicas ${ }^{(6)}$. Pesquisa realizada na Índia com 60 MPS, para detectar anormalidades em citologia vaginal, mostrou que, do total, 86,7\% dos esfregaços foram considerados anormais. E a incidência de lesão intraepitelial escamosa de alto grau foi de 38,3\%, considerada elevada na população do estudo ${ }^{(6)}$. Dessa forma, MPS estão em maior risco para a aquisição do câncer do colo do útero devido à alta exposição ao $\mathrm{HPV}^{(7)}$.

Diante da magnitude do problema, o Ministério da Saúde do Brasil recomenda, por meio da Linha de Cuidado do Câncer do Colo do Útero, o acesso humanizado e integral às ações e aos serviços qualificados para promover a prevenção do câncer do colo do útero, bem como o acesso ao rastreamento das lesões precursoras, ao diagnóstico precoce e ao tratamento adequado, qualificado e em tempo oportuno ${ }^{(3)}$. Recomenda-se ainda a necessidade de um acompanhamento rigoroso e exames histológicos, para evitar a disseminação desnecessária da doença 
neoplásica e a morte prematura entre as mulheres diagnosticadas ${ }^{(6)}$.

As MPS apresentam maior vulnerabilidade para as diversas infecções ginecológicas, considerando-se a dificuldade para receber orientações contextualizadas a seus estilos de vida e de modo que se sintam acolhidas em suas dúvidas acerca das formas de prevenção e de assistência, quando necessárias ${ }^{(8)}$.

Com base no exposto, o presente estudo teve como objetivos estimar a prevalência autorreferida da realização do exame citopatológico do colo do útero em mulheres profissionais do sexo e investigar a associação entre a realização desse teste e os dados socioeconômicos e comportamentais.

\section{Método}

Estudo analítico de corte transversal, desenvolvido com MPS em Teresina, capital do estado do Piauí, no Nordeste do Brasil, de janeiro de 2014 a fevereiro de 2015. Trata-se de um recorte de um macroprojeto com mulheres profissionais do sexo com financiamento do Ministério da Saúde, edital 01/2013 e carta acordo 130/2013.

Para a seleção das participantes, utilizou-se o método Respondent Drive Sampling (RDS), que inclui vários requisitos que são chave para a criação de uma amostra representativa. A escolha desse método justifica-se pelo fato de esse segmento ser organizado em rede e ser de difícil acesso. Essa metodologia é indicada para estudos com populações complexas, considerando que o acesso a esses grupos é mais fácil por intermédio de um membro deles ${ }^{(9)}$.

Para estimar o tamanho da amostra, foi considerada uma prevalência de 1,8\% da infecção por $\mathrm{HIV}^{(10)}$. Assim, estimaram-se, no mínimo, 384 participantes, sendo acrescentado o valor de 20\%, em virtude de eventuais perdas. O número final que compôs o estudo foi de 416 profissionais do sexo.

Foram adotados os seguintes critérios de inclusão: ter idade igual ou superior a 18 anos; trabalhar como MPS no município; ter tido pelo menos uma relação sexual em troca de dinheiro nos últimos quatro meses; apresentar um convite válido para participar do estudo. Como critério de exclusão adotou-se: estar visivelmente sob a influência de drogas, incluindo álcool, no momento da entrevista.

Para a coleta de dados, foram investigadas as características da população conforme o local de atuação, sendo utilizado um formulário estruturado validado com as seguintes questões: variáveis independentes - características sociodemográficas e econômicas (faixa etária, procedência, cor da pele autorreferida, estado civil, renda individual mensal, escolaridade, religião), acesso aos serviços de saúde e planejamento familiar (uso de método contraceptivo e uso de preservativo com parceiro fixo); e variáveis dependentes - realização do exame citopatológico do colo do útero (frequência de efetuação).

Para a determinação da prevalência de realização do exame preventivo para câncer do colo do útero, considerou-se a informação de efetuação do teste nos três anos anteriores à data da entrevista ${ }^{(3)}$.

Os dados foram digitados duplamente no Excel, em planilha eletrônica, e, após validação, foram exportados para o software $\operatorname{IBM}^{\mathrm{R}}$ SPSS 21.0. A análise das variáveis quantitativas foi apresentada em média, mínimos e máximos, e as categóricas foram exibidas em frequência. Para investigar a associação entre as variáveis, empregou-se o teste Qui-Quadrado, sendo considerada significância estatística em valor de p<0,005.

A pesquisa foi aprovada pelo Comitê de Ética em Pesquisa (CEP) da Universidade Federal do Piauí, sob o parecer n. 0425.0.045.000-11.

\section{Resultados}

Das 416 MPS, evidenciou-se maior proporção $(62,3 \%)$ naquelas com faixa etária de 25 a 39 anos (média de 30,48 anos; idade mínima de 18 e máxima de 59 anos), autodenominadas de cor branca (43,0\%), católicas (79,3\%), com escolaridade $>8$ anos de estudos (52,6\%), solteiras (82,0\%), com renda mensal entre 1 e 2 salários mínimos, cujo valor unitário à época do estudo era de $\mathrm{R} \$ 780,00$, equivalente a US\$333,00 (cotação em 15 de janeiro de 2014). (Tabela 1). 
Tabela 1 - Características sociodemográficas e econômicas de mulheres profissionais do sexo. Teresina, Piauí, Brasil - 2014-2015 (N=416)

\begin{tabular}{|c|c|c|}
\hline Variáveis & $\mathbf{n}$ & $\%$ \\
\hline \multicolumn{3}{|l|}{ Faixa etária } \\
\hline 18-24 anos & 104 & 25,0 \\
\hline 25-39 anos & 259 & 62,3 \\
\hline 40-59 anos & 53 & 12,7 \\
\hline \multicolumn{3}{|l|}{ Cor de pele autorreferida } \\
\hline Preta & 146 & 35,1 \\
\hline Parda & 87 & 20,9 \\
\hline Branca & 179 & 43,0 \\
\hline Amarela & 4 & 1,0 \\
\hline \multicolumn{3}{|l|}{ Estado civil } \\
\hline Solteira & 341 & 82,0 \\
\hline Casada & 22 & 5,3 \\
\hline Separada & 50 & 12,0 \\
\hline Viúva & 3 & 0,7 \\
\hline \multicolumn{3}{|c|}{ Renda mensal individual (Salário Mínimo) } \\
\hline Sem rendimento & 21 & 5,0 \\
\hline 1 a 2 & 174 & 41,9 \\
\hline 2 a 3 & 117 & 28,1 \\
\hline 4 a 10 & 104 & 25,0 \\
\hline \multicolumn{3}{|l|}{ Escolaridade } \\
\hline Analfabeta & 26 & 6,3 \\
\hline Ensino Fundamental incompleto & 219 & 52,6 \\
\hline Ensino Fundamental completo & 63 & 15,2 \\
\hline Ensino Médio incompleto & 58 & 13,9 \\
\hline Ensino Médio completo & 50 & 12,0 \\
\hline \multicolumn{3}{|l|}{ Religião } \\
\hline Nenhuma & 59 & 14,2 \\
\hline Católica & 330 & 79,3 \\
\hline Evangélica & 20 & 4,8 \\
\hline Outras & 7 & 1,7 \\
\hline
\end{tabular}

Fonte: Elaboração própria.

Em relação ao acesso ao serviço de saúde, 58,6\% das participantes referiram utilizar serviços públicos, nas proximidades de suas residências; 23,6\%, o serviço particular; $12 \%$ fazem contato com o Agente Comunitário de Saúde (ACS) e 5,8\% não buscam assistência à saúde. Quanto às medidas de contracepção e proteção, 69,0\% informaram não utilizar nenhum método contraceptivo. Em relação ao preservativo, do total de MPS que informaram ter parceria fixa, 51,5\% informaram nunca usar com esse tipo de parceria.
No tocante ao exame citopatológico do colo do útero, 15,1\% nunca o realizaram. A prevalência autorreferida de realização do exame citopatológico do colo do útero foi de 47,8\%. Do total de MPS que fizeram o teste, $60,1 \%$ utilizaram unidades de saúde pública, 4,1\% o realizaram em ônibus, em campanhas educativas, 18,5\%, em clínicas privadas e 17,3\% não responderam (Tabela 2). 
Tabela 2 - Características do planejamento familiar e do acesso aos serviços de saúde por mulheres profissionais do sexo. Teresina, Piauí, Brasil - 2014-2015 (N=416)

\begin{tabular}{|c|c|c|}
\hline Variáveis & $\mathbf{n}$ & $\%$ \\
\hline \multicolumn{3}{|l|}{ Acesso ao serviço de saúde } \\
\hline Consultas nas proximidades da residência & 244 & 58,6 \\
\hline Agente Comunitário de Saúde & 50 & 12,0 \\
\hline Serviço privado & 98 & 23,6 \\
\hline Não utiliza o serviço de saúde & 24 & 5,8 \\
\hline \multicolumn{3}{|l|}{ Uso de contraceptivo } \\
\hline $\operatorname{Sim}$ & 128 & 30,8 \\
\hline Não & 287 & 69,0 \\
\hline Não respondeu & 1 & 0,2 \\
\hline \multicolumn{3}{|c|}{ Uso de preservativo com parceiro fixo $(n=200)$} \\
\hline Nunca & 103 & 51,5 \\
\hline Às vezes & 26 & 13,0 \\
\hline Sempre & 1 & 0,5 \\
\hline Não respondeu & 70 & 35,0 \\
\hline \multicolumn{3}{|c|}{ Tempo de realização do citopatológico do colo do útero } \\
\hline Nunca & 63 & 15,1 \\
\hline Menos de 1 ano & 68 & 16,3 \\
\hline $1-3$ anos & 131 & 31,5 \\
\hline Mais de 3 anos & 102 & 24,6 \\
\hline Mais de 5 anos & 51 & 12,3 \\
\hline Não respondeu & 1 & 0,2 \\
\hline \multicolumn{3}{|l|}{ Local de realização do exame } \\
\hline Unidade de saúde pública & 250 & 60,1 \\
\hline Em ônibus & 17 & 4,1 \\
\hline Clínica privada & 77 & 18,5 \\
\hline Não respondeu & 72 & 17,3 \\
\hline
\end{tabular}

Fonte: Elaboração própria.

Houve associação estatística entre a realização do exame citopatológico do colo do útero e as seguintes variáveis: renda $(\mathrm{p}=0,001)$, escolaridade $(\mathrm{p}=0,001)$, religião $(\mathrm{p}=0,001)$, acesso ao serviço $(\mathrm{p}=0,003)$ e uso de contraceptivo $(\mathrm{p}=0,003)$. (Tabela 3).

Tabela 3-Medidas de associação entre as características sociodemográficas, econômicas, planejamento familiar e de acesso aos serviços de saúde de mulheres profissionais do sexo e a realização do exame citopatológico do colo do útero. Teresina, Piauí, Brasil - 2014-2015 (N=416)

(continua)

\begin{tabular}{|c|c|c|c|c|c|}
\hline \multirow{3}{*}{ Variável } & & \multirow{3}{*}{$P^{*}$} \\
\hline & \multicolumn{4}{|c|}{ Sim } & \\
\hline & $\mathbf{n}$ & $\%$ & $\mathbf{n}$ & $\%$ & \\
\hline \multicolumn{6}{|c|}{ Faixa etária (anos) } \\
\hline $18-24$ & 82 & 19,7 & 22 & 5,3 & \\
\hline $25-39$ & 224 & 53,8 & 35 & 8,5 & 0,131 \\
\hline $40-59$ & 47 & 11,3 & 06 & 1,4 & \\
\hline \multicolumn{6}{|c|}{ Cor de pele autorreferida } \\
\hline Preta & 126 & 30,3 & 20 & 4,8 & \\
\hline Parda & 66 & 15,9 & 21 & 5,0 & 0,056 \\
\hline Branca & 157 & 37,8 & 22 & 5,3 & \\
\hline Amarela & 04 & 0,9 & 00 & 0,0 & \\
\hline
\end{tabular}


Tabela 3-Medidas de associação entre as características sociodemográficas, econômicas, planejamento familiar e de acesso aos serviços de saúde de mulheres profissionais do sexo e a realização do exame citopatológico do colo do útero. Teresina, Piauí, Brasil - 2014-2015 (N=416)

(conclusão)

\begin{tabular}{|c|c|c|c|c|c|}
\hline \multirow{3}{*}{ Variável } & \multicolumn{4}{|c|}{ Realização do citopatológico do colo do útero } & \multirow{3}{*}{$P^{*}$} \\
\hline & \multicolumn{4}{|c|}{\begin{tabular}{|c|c|} 
Sim & Não
\end{tabular}} & \\
\hline & $\mathbf{n}$ & $\%$ & $\mathbf{n}$ & $\%$ & \\
\hline \multicolumn{6}{|l|}{ Estado civil } \\
\hline Solteira & 289 & 69,5 & 52 & 12,5 & \multirow{4}{*}{0,862} \\
\hline Casada & 18 & 4,2 & 04 & 0,9 & \\
\hline Separada & 43 & 10,4 & 07 & 1,7 & \\
\hline Viúva & 03 & 0,8 & 00 & 0,0 & \\
\hline \multicolumn{6}{|c|}{ Renda mensal individual (Salário Mínimo) } \\
\hline Sem rendimento & 09 & 2,2 & 12 & 2,9 & \multirow{4}{*}{$\mathbf{0 , 0 0 1}$} \\
\hline 1 a 2 & 132 & 31,7 & 42 & 10,1 & \\
\hline 2 a 3 & 112 & 26,9 & 05 & 1,2 & \\
\hline 4 a 10 & 100 & 24,0 & 04 & 1,0 & \\
\hline \multicolumn{6}{|l|}{ Escolaridade } \\
\hline Analfabeta & 19 & 4,6 & 07 & 1,7 & \multirow{5}{*}{0,001} \\
\hline Ensino Fundamental incompleto & 182 & 43,8 & 37 & 8,9 & \\
\hline Ensino Fundamental completo & 58 & 13,9 & 05 & 1,2 & \\
\hline Ensino Médio incompleto & 50 & 12,0 & 08 & 1,9 & \\
\hline Ensino Médio completo & 44 & 10,6 & 06 & 1,4 & \\
\hline \multicolumn{6}{|l|}{ Religião } \\
\hline Nenhuma & 51 & 12,3 & 08 & 1,9 & \multirow{4}{*}{0,001} \\
\hline Católica & 276 & 66,4 & 54 & 12,9 & \\
\hline Evangélica & 19 & 4,6 & 01 & 0,2 & \\
\hline Outras & 07 & 1,7 & 00 & 0,0 & \\
\hline \multicolumn{6}{|l|}{ Acesso ao serviço de saúde } \\
\hline Unidade de Saúde Básica & 210 & 50,5 & 34 & 8,2 & \multirow{4}{*}{0,003} \\
\hline Agente Comunitário de Saúde & 36 & 8,6 & 14 & 3,4 & \\
\hline Serviço privado & 90 & 21,6 & 08 & 1,9 & \\
\hline Não utiliza o serviço de saúde & 17 & 4,1 & 07 & 1,7 & \\
\hline \multicolumn{6}{|l|}{ Uso de contraceptivo $(n=415)$} \\
\hline $\operatorname{Sim}$ & 119 & 28,7 & 09 & 2,2 & \multirow[t]{2}{*}{0,003} \\
\hline Não & 234 & 56,3 & 53 & 12,8 & \\
\hline \multicolumn{6}{|c|}{ Uso de preservativo com parceiro fixo $(n=200)$} \\
\hline Nunca & 84 & 42,0 & 19 & 9,5 & \multirow{4}{*}{0,139} \\
\hline Às vezes & 23 & 11,5 & 03 & 1,5 & \\
\hline Sempre & 00 & 0,0 & 01 & 0,5 & \\
\hline Não recorda & 55 & 27,5 & 15 & 7,5 & \\
\hline
\end{tabular}

Fonte: Elaboração própria.

* Referente à realização do teste do Qui-quadrado: diferença estatisticamente significante (p-valor $\leq 0,05)$.

\section{Discussão}

Neste estudo, as MPS apresentaram baixa prevalência para a realização do exame citopatológico do colo do útero (47,8\%) quando comparadas com as de outra capital do Nordeste do Brasil $(95,1 \%)^{(11)}$. Isso se deu também na comparação com demais mulheres da população de um município de Boa Vista, região Norte do Brasil, que foi de $85,7 \%$ em 517 participantes ${ }^{(12)}$.

A realização do exame citopatológico do colo do útero varia conforme as características individuais e regionais. Neste estudo, em relação à faixa etária, MPS com 18 e 24 anos e 40 e 59 
anos realizaram o exame preventivo com menor frequência, sendo $19,7 \%$ e $11,3 \%$, respectivamente. Um estudo realizado em uma capital do Nordeste do Brasil, com 250 MPS, mostrou que a maioria $(61,2 \%)$ que realizou o exame colpocitológico tinha em média 35 anos de idade ${ }^{(13)}$.

Nesta pesquisa, as MPS solteiras apresentaram maior taxa de efetuação do exame. Estudos mostraram que tanto as mulheres solteiras com maior multiplicidade de parceiros sexuais quanto as casadas, quando não usam o preservativo em todas as relações sexuais, estão mais expostas às IST $^{(12,14)}$. A não realização do exame é preocupante, independente do estado conjugal.

Baixa escolaridade $(\mathrm{p}=0,001)$ e baixa renda $(\mathrm{p}=0,001)$ foram identificadas como estatisticamente significativas para a não realização do exame citopatológico do colo do útero. Um estudo realizado no interior do Piauí, Nordeste do Brasil, com 77 MPS mostrou que 47 (61\%) tinham Ensino Fundamental incompleto; quando questionadas sobre o exame preventivo, 30 (38,9\%) não souberam informar a finalidade ${ }^{(15)}$.

Em relação à cor da pele, preta/parda foram fatores relacionados à não realização do citopatológico do colo do útero. Quanto ao conhecimento sobre a finalidade do teste, mulheres de cor não branca apresentaram associação para a não efetuação $(p=0,019)^{(16)}$. Autores sugerem que a não efetuação preventiva do citopatológico do colo do útero pode ser justificada pelo baixo nível de conhecimento sobre as infecções e os seus efeitos prejudiciais à saúde ${ }^{(17-18)}$.

Um estudo realizado na região Norte do Brasil, com mulheres da população, mostrou que a etnia e a religiosidade não tiveram relevância estatística para a não realização do exame e que, quanto ao local, foi relatado que as igrejas são preferenciais, por proporcionarem melhor acolhimento. O estudo ressaltou ainda que estratégias nesse sentido poderiam ser desenvolvidas para aumentar as taxas de cobertura do exame ${ }^{(19)}$.

A maioria das MPS buscou acesso ao serviço de saúde pública, para a realização do exame citopatológico do colo do útero, e houve significância estatística. No Brasil, a Estratégia Saúde da Família (ESF) contribui para a redução dos indicadores de morbimortalidade, considerado um modelo de atenção à saúde norteado pelos princípios do Sistema Único de Saúde (SUS). O ACS, membro da equipe, pode contribuir para a superação das barreiras existentes à realização do exame, quando identifica e captura as mulheres que deixaram de realizá-lo ${ }^{(20)}$.

Apesar de a maioria das MPS terem buscado as unidades públicas de saúde com maior frequência, o estudo mostrou que um percentual elevado das participantes não atendeu a recomendação da Organização Mundial de Saúde (OMS) para a realização do exame, que deve ocorrer a cada três anos, após dois testes normais consecutivos, com intervalo de um ano ${ }^{(19)}$. Um estudo realizado na região Sudeste do Brasil, com demais mulheres da população, mostrou que ter história prévia de três ou mais colpocitologias proporciona uma proteção de 84\% (OR=0,16; IC95\%: 0,07; 0,38) para o câncer cervical uterino e que o setor público é o mais utilizado pelas mulheres para rastreamento de câncer do colo do útero ${ }^{(21)}$.

Estudo mostrou que o intervalo entre a data do resultado da citologia e a data da primeira colposcopia, entre aquelas mulheres que obtiveram teste do citopatológico do colo do útero alterado, foi considerado longo, com média de espera de 94,5 dias. Mostrou ainda que devem ser adotadas medidas para reduzir o tempo de espera do resultado do primeiro exame para a realização da segunda colposcopia, de modo a melhorar a qualidade da assistência no âmbito do controle do câncer do colo do útero ${ }^{(22)}$. Entretanto, a baixa cobertura do exame possivelmente está associada à busca ativa insuficiente e à dificuldade de agendamento das consultas na atenção básica, bem como às dificuldades de acesso e ao atendimento insatisfatório ${ }^{(19)}$.

Embora o risco do câncer do colo uterino seja alto em todas as mulheres, as MPS estão mais expostas à infecção por HPV, como demonstra estudo realizado no Peru, com 200 participantes, ao identificar altas taxas de transmissão do HPV, destacando a necessidade de estratégias precoces e eficazes para prevenir o câncer do colo do útero nessa população ${ }^{(23)}$. Acrescenta-se que 
resultado de citologia anormal foi encontrado também na Tailândia, em 10\% das MPS e em $5 \%$ das demais mulheres. Desse total, o HPV foi detectado em 13\% das MPS e em 9\% das demais mulheres ${ }^{(24)}$.

Uma das medidas preconizadas para a prevenção de IST é o uso de preservativos. É considerado essencial para a interrupção da cadeia de transmissão, inclusive para a prevenção de neoplasias relacionadas às atividades sexuais desprotegidas, como o câncer do colo do útero associado ao HPV, e também como método contraceptivo. Pesquisa realizada em uma capital do Nordeste brasileiro, com mulheres de 25 a 59 anos, mostrou que o não uso de método contraceptivo foi significativamente associado à não adesão ao exame citopatológico do colo do útero. Dessa forma, tem-se um risco duplicado de infecção e posterga-se o diagnóstico precoce para certas neoplasias ${ }^{(25)}$.

Neste estudo, dentre as $47,8 \%$ das mulheres que relataram ter realizado o exame, $28,7 \%$ faziam uso de algum método contraceptivo. Isso sugere que é fundamental a criação de espaços onde as MPS possam ter acesso adequado às orientações e aos insumos. O uso de algum método contraceptivo pode constituir um fator de proteção para a realização do referido procedimento preventivo.

Em relação ao uso do preservativo com a parceria fixa, 103 (51,5\%) mulheres informaram que nunca utilizam; desse total, 19 (9,5\%) admitiram não ter realizado exame citopatológico do colo do útero. Pesquisa revelou que menos da metade das MPS $(47,7 \%)$ usam preservativos com qualquer tipo de parceiro, 71,9\% usam com a parceria fixa e $84,2 \%$, com parceria casual $^{(26)}$. Entre as principais barreiras elencadas para o não uso desse método preventivo, têm-se: pobreza, recusa de usar preservativos por parceiros do sexo masculino, uso de álcool antes do sexo e crenças de que esses métodos reduzem o prazer sexual $^{(27)}$.

Os achados desta pesquisa têm como limitações: a questão sobre a realização do exame citopatológico do colo do útero basear-se em lembranças (viés de memória); o fato de não ser possível generalizar os achados ao total da população de MPS, por ser esse um segmento de amostragem com maiores estigmas sociais e de acesso limitado. Além disso, trata-se do delineamento de natureza transversal, não sendo possível o estabelecimento de relações de causa e efeito. Sugere-se que novas pesquisas sejam realizadas com essa população, tendo como foco o rastreamento de câncer do colo uterino e orientações sobre a redução de risco.

\section{Conclusão}

Neste estudo, mulheres profissionais do sexo apresentaram baixa adesão autorreferida de realização do exame citopatológico do colo do útero, quando comparadas a populações semelhantes de outra capital e também às demais mulheres da população. Os principais fatores associados para a sua realização foram a renda, a escolaridade, a religião, bem como as formas de acesso ao serviço de atendimento e o uso de contraceptivo.

Em face do supracitado, faz-se necessária a realização de ações de saúde integradas para essa população. Recomenda-se a melhoria do acesso das mulheres profissionais do sexo a serviços públicos de saúde, com vistas à realização de atividades de educação em saúde, considerando suas características individuais, para ampliação do conhecimento sobre medidas de prevenção do câncer do colo do útero. Nesse contexto, considerando a maior vulnerabilidade das MPS, urge a necessidade de ampliar oportunidades de rastreamento de casos de câncer do colo do útero para populações semelhantes.

\section{Colaborações:}

1. concepção, projeto, análise e interpretação dos dados: Rosilane de Lima Brito Magalhães, Braulio Vieira de Sousa Borges, Vanessa Moura Carvalho de Oliveira, Giselle Mary Ibiapina Brito e Ana Karysa Alves Resende;

2. redação do artigo e revisão crítica relevante do conteúdo intelectual: Rosilane de Lima Brito Magalhães, Braulio Vieira de Sousa 
Borges, Vanessa Moura Carvalho de Oliveira e Giselle Mary Ibiapina Brito;

3. aprovação final da versão a ser publicada: Elucir Gir.

\section{Referências}

1. ICO/IARC Information Centre on HPV and Cancer. Brazil. Human Papillomavirus and related cancers, fact sheet 2017 [Internet]. Barcelona (ESP); 2017.

2. Guerra MR, Bustamante-Teixeira MT, Corrêa CSL, Abreu DMX, Curado MP, Mooney M, et al. Magnitude e variação da carga da mortalidade por câncer no Brasil e Unidades da Federação, 1990 e 2015. Rev bras epidemiol [Internet]. 2017 May [cited 2018 Sep 7];20(Suppl 1):102-15. Available from: http://www.scielo.br/scielo.php?script $=$ sci arttext\&pid=S1415-790X2017000500102\&lng=en. http://dx.doi.org/10.1590/1980-5497201700050009

3. Brasil. Ministério da Saúde. Secretaria de Atenção à Saúde. Departamento de Atenção Básica. Controle dos cânceres do colo do útero e da mama. 2a ed. Brasília; 2013.

4. Spice W. Management of sex workers and other high-risk groups. Occup Med (Lond) [Internet]. 2007 Aug [cited 2018 Jan 12];57:322-8. Available from: https://academic.oup.com/occmed/article/ $57 / 5 / 322 / 1406166$

5. Jia H, Wang X, Long Z, Li L. Human papillomavirus infection and cervical dysplasia in female sex workers in Northeast China: an observational study. BMC Public Health [Internet]. 2015 July [cited 2018 Jan 12];15(695):1-6. Available from: https://www. ncbi.nlm.nih.gov/pmc/articles/PMC4512111/ pdf/12889_2015_Article_2066.pdf

6. Mondal SK, Basak B, Roy DN, Mandal PK, Sinha S. Evaluation of vaginal cytology in female sex-workers: A study in a tertiary hospital of eastern India. J Cytol [Internet]. 2014 [cited 2018 Sep 7]:31(1):7-10. Available from: https://www. ncbi.nlm.nih.gov/pmc/articles/PMC4150348/

7. Soohoo M, Blas M, Byraiah G, Carcamo C, Brown B. Cervical HPV Infection in Female Sex Workers: a global perspective. Open AIDS J [Internet]. 2013 [cited 2018 Sep 7];7:58-66.

8. Silva RM, Araújo MAL, Pessoa CM, Moraes MP. Saberes e práticas de prostitutas acerca dos métodos contraceptivos. Rev baiana saúde pública [Internet]. 2008 [cited 2018 Feb 6];32(2):177-89.
Available from: http://rbsp.sesab.ba.gov.br/index. $\mathrm{php} / \mathrm{rbsp} /$ article/view/1430/1067

9. Barbosa Júnior A, Szwarcwald CL, Pascom ARP, Souza Júnior PB. Tendências da epidemia de AIDS entre subgrupos sob maior risco no Brasil, 19802004. Cad Saúde Pública [Internet]. 2009 [cited 2018 Feb 6];25(4):727-37. Available from: http:// www.scielo.br/pdf/csp/v25n4/03.pdf

10. Caetano KA, França DD, Carneiro MA, Martins RM, Stefani MM, Kerr LR, et al. Prevalence and virologic profile of HIV infections among female sex workers in Goiânia City, central Brazil. AIDS Patient Care STDS [Internet]. 2013 [cited 2018 Feb 6];27(1):1-4. Available from: https://www.liebertpub.com/doi/ abs/10.1089/apc.2012.0268?journalCode=apc

11. Nicolau AIO, Aquino PS, Moura ERF, Pinheiro AKB. Perfil gineco-obstétrico e realização do exame de prevenção por prostitutas de Fortaleza. Rev RENE [Internet]. 2008 [cited 2018 Feb 6];9(1):103-10. Available from: http://www.periodicos.ufc.br/ rene/article/view/5008/3686

12. Navarro C, Fonseca AJ, Sibajev A, Souza CIA, Araújo DS, Teles DAF, et al. Cervical cancer screening coverage in a high-incidence region. Rev Saúde Pública [Internet]. 2015 [cited 2018 Feb 6];49(17);1-8. Available from: http://www.scielo.br/pdf/rsp/ v49/0034-8910-rsp-S0034-89102015049005554.pdf

13. Vasconcelos CTM, Pinheiro AKB, Castelo ARP, Costa LQ, Oliveira RG. Conhecimento, atitude e prática relacionada ao exame colpocitológico entre usuárias de uma unidade básica de saúde. Rev Latino-Am Enfermagem [Internet]. 2011 [cited 2018 Sep 12];19(1):97-105. Available from: http:// www.scielo.br/pdf/rlae/v19n1/pt_14.pdf

14. Gasperin SI, Boing AF, Kupek E. Cobertura e fatores associados à realização do exame de detecção do câncer de colo de útero em área urbana no Sul do Brasil: estudo de base populacional. Cad Saúde Pública [Internet]. 2011 [cited 2018 Feb 6];27(7):1312-22. Available from: http://www. scielo.br/pdf/csp/v27n7/07.pdf

15. Neri ÉAR, Moura MSS, Penha JC, Reis TGO, Aquino PS, Pinheiro AKB. Conhecimento, atitude e prática sobre o exame papanicolaou de prostitutas. Texto contexto-enferm [Internet]. 2013 [cited 2018 Sep 11];22(3):731-8. Available from: http://dx.doi. org/10.1590/S0104-07072013000300020

16. Rodrigues Neto JF, Figueiredo MFS, Siqueira LG. Exame citopatológico do colo do útero: fatores associados a não realização em ESF. Rev Eletr Enf 
[Internet]. 2008 [cited 2018 Feb 6];10(3):610-21. Available from: http://www.fen.ufg.br/revista/v10/ n3/v10n3a07.htm

17. Silva DSM, Silva AMN, Brito LMO, Gomes SRL, Nascimento MDSB, Chein MBC. Rastreamento do câncer do colo do útero no Estado do Maranhão, Brasil. Ciênc Saúde Coletiva [Internet]. 2014 [cited 2018 Jan 15];19(4):1163-70. Available from: http://www.scielo.br/pdf/csc/v19n4/14138123-csc-19-04-01163.pdf

18. Sabeena S, Bhat PV, Kamath V, Aswathyraj S, Arunkumar G. Knowledge, Attitude and Practice Concerning Human Papilloma Virus Infection and its Health Effects among Rural Women, Karnataka, South India. Asian Pac J Cancer Prev [Internet]. 2015 [cited 2018 Mar 6];16(12):5053-8. Available from: http://journal. waocp.org/article_31211_1144da3327d6555e9411 fc30e5d0e974.pdf

19. Lucena LT, Zãn DG, Crispim PTB, Ferrari JO. Fatores que influenciam a realização do exame preventivo do câncer cérvico-uterino em Porto Velho, Estado de Rondônia, Brasil. Rev Pan-Amaz Saude [Internet]. 2011 [cited 2018 Feb 6];2(2):45-50. Available from: http://scielo.iec.gov.br/pdf/rpas/ v2n2/v2n2a07.pdf

20. Bousso RS, Silva L, Lima AN. A Visita Domiciliária Realizada pelo Agente Comunitário de Saúde sob a ótica de adultos e idosos. Saúde Soc São Paulo [Internet]. 2010 [cited 2018 Sep 12];19(4):889-97. Available from: http://www.scielo.br/pdf/sausoc/ v19n4/15.pdf

21. Girianelli VR, Thuler LCS, Silva GA. Adesão ao rastreamento para câncer do colo do útero entre mulheres de comunidades assistidas pela Estratégia Saúde da Família da Baixada Fluminense, Rio de Janeiro, Brasil. Rev Bras Ginecol Obstet [Internet]. 2014 [cited 2018 Feb 6];36(5):198-204. Available from: http://www.scielo.br/pdf/rbgo/v36n5/01007203-rbgo-36-05-00198.pdf

22. Nascimento MI, Rabelo IMMA, Cardoso FSP, Musse RNV. Tempo de espera pela primeira colposcopia em mulheres com teste de Papanicolaou alterado. Rev Bras Ginecol Obstet [Internet]. 2015 [cited 2018 Feb 6];37(8):381-7. Available from: http:// www.scielo.br/pdf/rbgo/v37n8/0100-7203rbgo-37-08-00381.pdf

23. Brown B, Blas MM, Cabral A, Byraiah G, GuerraGiraldez C, Sarabia-Vega V, et al. Human papillomavirus prevalence, cervical abnormalities and risk factors among female sex workers in Lima, Peru. Int J STD AIDS [Internet]. 2012 [cited 2018 Mar 6];23(4):242-7. Available from: https://www. ncbi.nlm.nih.gov/pmc/articles/PMC4699584/pdf/ nihms680390.pdf

24. Leaungwutiwong P, Bamrungsak B, Jittmittraphap A, Maneekan P, Kosoltanapiwat N, Kalambaheti T, et al. Molecular genotyping of human papillomavirus 11 gene in low-risk and high-risk populations in Bangkok. Sex Transm Dis [Internet]. 2015 [cited 2018 Feb 6];42(4):208-17. Available from: https://www.ncbi.nlm.nih.gov/pmc/articles/ PMC4358745/pdf/nihms661979.pdf

25. Andrade MS, Almeida MMG, Araújo TM, Santos KOB. Fatores associados a não adesão ao Papanicolau entre mulheres atendidas pela Estratégia Saúde da Família em Feira de Santana, Bahia, 2010. Epidemiol Serv Saúde [Internet]. 2014 [cited 2018 Feb 6];23(1):111-20. Available from: http://www.scielo.br/pdf/ress/v23n1/2237-9622ress-23-01-00111.pdf

26. Tamene MM, Tessema GA, Beyera GK. Condom utilization and sexual behavior of female sex workers in Northwest Ethiopia: a cross-sectional study. Pan Afr Med J [Internet]. 2015 [cited 2018 Feb 6];21(50):1-10. Available from: https://www. ncbi.nlm.nih.gov/pmc/articles/PMC4564417/pdf/ PAMJ-21-50.pdf

27. Matovu JKB, Ssebadduka NB. Knowledge, attitudes \& barriers to condom use among female sex workers and truck drivers in Uganda: a mixed-methods study. Afr Health Sci [Internet]. 2013 [cited 2018 Feb 6];13(4):1027-33. Available from: https://www.ncbi.nlm.nih.gov/pmc/articles/ PMC4056494/pdf/AFHS1304-1027.pdf

Recebido: 1 de março de 2018

Aprovado: 25 de julho de 2018

Publicado: 27 de novembro de 2018 
A Revista Baiana de Enfermagem utiliza a Licença Creative Commons - Atribuição-NãoComercial 4.0 Internacional.

https://creativecommons.org/licenses/by-nc/4.0/

Este artigo é de acesso aberto distribuído sob os termos da Licença Creative Commons (CC BY-NC).

Esta licença permite que outros remixem, adaptem e criem a partir do seu trabalho para fins não comerciais.

Embora os novos trabalhos tenham de lhe atribuir o devido crédito e não possam ser usados para fins comerciais, os usuários não têm de licenciar esses trabalhos derivados sob os mesmos termos. 